\title{
Recent advances in multimodal treatment for gastric cancer: a review
}

\author{
Florian LORDick and J. RÜDIGER SiewerT \\ Klinikum rechts der Isar, Technical University of Munich, Ismaninger Str. 22, D-81675 Munich, Germany
}

\begin{abstract}
The prognosis after surgical treatment of gastric cancer remains poor. This review aims to clarify the indication for multimodal treatment of gastric cancer. A systematic literature research and evaluation of clinical data presented at important international cancer meetings were undertaken. Recently published results of large randomized phase III trials underscore the potential value of neoadjuvant chemotherapy or adjuvant chemoradiotherapy in gastric cancer. However, data from randomized controlled trials are still scarce, and it remains uncertain which subgroups of patients should routinely undergo multimodal treatment. In view of the side effects of pre- and foremost postoperative therapies, a more distinct definition of prognostic and predictive markers is warranted. Neoadjuvant chemotherapy is a rising option in locally advanced gastric cancer. Adjuvant chemoradiation has been shown to be beneficial in gastric cancer patients who have undergone suboptimal surgical resection. The benefits of adjuvant chemotherapy alone seem to be very small.
\end{abstract}

Key words Gastric cancer - Multimodal - Neoadjuvant · Adjuvant $\cdot$ Chemotherapy $\cdot$ Radiotherapy

\section{Introduction}

Important advances in the surgical and nonsurgical treatment of upper gastrointestinal (GI) cancer have been achieved during the last decades. However, the prognosis of gastric cancer remains poor. At least in the Western world, where esophagogastroduodenoscopy is not yet part of screening programs, upper GI tumors are commonly diagnosed in advanced stages [1]. Apart from that fact, lymphatic and hematogenous spread is often present even in early stages. Moreover, gastric cancer is only moderately sensitive to chemotherapy and radiation therapy. For this reason, the potential role of neoadjuvant and adjuvant treatment remained

Offprint requests to: $\mathrm{F}$. Lordick

Received: February 14, 2005 / Accepted: February 22, 2005 uncertain. Now, with the presentation of the preliminary survival results of the MRC Adjuvant Gastric Infusional Chemotherapy "MAGIC" trial [2], there is rising evidence that patients may benefit from neoadjuvant chemotherapy. On the other hand, the publication of the Southwest Oncology Group 9008/ Intergroup 0116 (SWOG9008/INT0116) trial [3] has defined adjuvant chemoradiation as a new standard of care in some countries. Hence, the interest in the role of combined-modality treatment in gastric cancer has rapidly increased among all physicians who care for gastric cancer patients. This review aims to clarify the current role of neoadjuvant and adjuvant treatment options for gastric cancer.

\section{Surgical resection}

Whenever one aims to define the role of multimodal therapy in nonmetastatic gastric cancer, one should bear in mind that surgical resection remains the mainstay of care in this disease. Unskilled surgery does reduce the chance for cure. Neither neoadjuvant nor adjuvant treatment can fully compensate for suboptimal surgical results. After years of debate on the extent of resections, recently published data help to settle the controversy. Briefly, for gastric carcinoma there is increasing confirmation of the superiority of an extended (D2) lymphadenectomy over a limited (D1) lymphadenectomy provided that splenectomy and distal pancreatectomy are omitted and that extended lymphadenectomy can be carried through with acceptable perioperative mortality and morbidity [4-9]. In adenocarcinoma of the esophagogastric junction type II and type III, extended total gastrectomy with transhiatal resection of the distal esophagus and D2 lymphadenectomy is the recommended procedure [10-13].

However, even after extended surgery carried through at experienced centers, long-term survival, at 
least in the "Western world," does not exceed approximately $50 \%[4,7]$. It is noteworthy that in patients with metastatic disease and patients in whom no complete resection can be achieved, long-term survival after surgery is very rare [7]. Therefore, as a general rule, patients with low probability for a complete resection (R0) should not be referred to primary surgery. Thus, for accurate treatment planning, particularly with regard to stage-dependent selection of neoadjuvant therapies, the most accurate clinical TNM stage must be worked out for patients scheduled for surgical resection.

\section{Staging procedures prior to multimodal treatment}

Endoscopic ultrasound (EUS) has proved to be superior to other methods in the prediction of pathologically confirmed $\mathrm{T}$ category with an accuracy of $79 \%-92 \%$ [14]. The clinical assessment of lymph node involvement is difficult with currently available preoperative staging techniques. Therefore, $\mathrm{N}$-stage often remains uncertain after the clinical staging. As a consequence, inclusion of patients into neoadjuvant treatment studies may preferentially be based on the findings from the $\mathrm{T}$ category assessed by EUS. The accuracy of EUS to predict R0 resectability has been evaluated as being $85 \%-90 \%[15,16]$.

Incipient peritoneal carcinomatosis cannot be detected by noninvasive techniques. Hence, although under debate for primary surgery [17], laparoscopy should be performed in any patient who is to undergo neoadjuvant treatment within clinical studies in order to achieve the most accurate information on the pretreatment tumor stage [18]. In addition, with the availability of staging laparoscopy, cytological examination of abdominal lavage fluid can be carried through. Positive findings are associated with poor prognosis [19-21].

Consequently, before a decision on multimodal treatment is to be taken, staging procedures may comprise endoscopic ultrasound to define T category, computed tomography to exclude distant metastases, and laparoscopy to exclude peritoneal carcinomatosis. The value of fluorodeoxyglucose (FDG) positron emission tomography (PET) is limited in gastric cancer as its sensitivity is as low as $41 \%-63 \%$ in nonintestinal type histology according to the Lauren classification $[22,23]$.

\section{Rationale for neoadjuvant and adjuvant therapy}

Multimodal approaches are based either on radiotherapy, which basically may increase the local tumor control, and/or chemotherapy in order to improve the systemic disease control. Both may be applied pre- or postoperatively, which is usually called neoadjuvant and adjuvant, respectively. Before going into a more detailed discussion of the pivotal clinical study results, three more general aspects merit some attention.

\section{Feasibility}

After a radical tumor resection in the upper gastrointestinal tract, the potential effectiveness of adjuvant treatment is often hampered by poor tolerance. This is mainly due to the postoperatively impaired physical condition of the patients. Body-weight loss during the first 6 months after gastrectomy approximates $10 \%$ in relation to the preoperative body weight [24]. As a consequence, patients may encounter an increased rate and intensity of side effects. Neoadjuvant treatment strategies, on the other hand, have proved to be feasible and well tolerated by patients in different phase II and phase III trials. Results of the recently presented MAGIC trial have shown that $88 \%$ of patients completed three cycles of preoperative chemotherapy, whereas only $55 \%$ of patients started with postoperative chemotherapy and only $40 \%$ were able to receive all six planned perioperative cycles of chemotherapy [2].

\section{Local and systemic effects}

Neoadjuvant treatment may, theoretically, be superior to postoperative therapy for two further reasons. First, neoadjuvant treatment potentially leads to "downsizing" of the tumor and therefore may improve the complete resectability rate, which is the cornerstone of cure in oncological surgery. Second, neoadjuvant induction chemotherapy is the earliest way of tackling the systemic spread of cancer cells, which in many cases is the cause of the poor long-term prognosis [25]. Studies evaluating the pattern of recurrence after curative gastrectomy revealed that in only $30 \%-38 \%$ of patients a sole locoregional failure was found, whereas the remainder of the patients encountered distant or combined local and distant failure $[4,26]$.

\section{Follow-up during neoadjuvant therapy}

Owing to progress in investigational techniques, the accuracy of clinical staging has certainly improved. On the other hand, monitoring during neoadjuvant treatment and accurate response evaluation are still entirely unsatisfactory. With the exception of bulky lymph node involvement [27], clinical response evaluation criteria frequently used for metastatic disease have not been validated for localized tumors limited to the gastric wall [28]. Endoscopic ultrasound, although accurate in assessing $\mathrm{T}$ category in patients who have not been pretreated, cannot be relied on in patients who have 
Table 1. Neoadjuvant chemotherapy for gastric cancer: results of phase II and small phase III studies

\begin{tabular}{|c|c|c|c|c|c|}
\hline $\begin{array}{l}\text { Study } \\
\text { (First author; ref. \#) }\end{array}$ & Regimen & $\begin{array}{c}\text { No. } \\
\text { patients }\end{array}$ & $\begin{array}{c}\text { Resectable } \\
\text { (R0) no. (\%) }\end{array}$ & $\begin{array}{l}\text { Median } \\
\text { survival }\end{array}$ & $\begin{array}{l}\text { 2-year } \\
\text { survival }\end{array}$ \\
\hline Wilke 1989 [31] & EAP & 34 & $10(29 \%)$ & $18 \mathrm{mo}$ & $26 \%$ \\
\hline Ajani 1991 [32] & EFP & 25 & $18(72 \%)$ & $15 \mathrm{mo}$ & $44 \%$ \\
\hline Alexander 1993 [33] & FU-FA-INFalfa2a & 22 & $18(82 \%)$ & $18 \mathrm{mo}$ & $52 \%$ \\
\hline Kelsen 1996 [29] & $\begin{array}{l}\text { pre-op. FAMTX } \\
\text { post-op. ip. } \\
\text { CDDP/FU; iv. FU }\end{array}$ & 56 & $34(61 \%)$ & $15 \mathrm{mo}$ & $40 \%$ \\
\hline Crookes 1997 [34] & $\begin{array}{l}\text { CDDP-FU } \\
\text { post-op. ip. CTx }\end{array}$ & 59 & $40(68 \%)$ & $>4 \mathrm{yr}$ & $64 \%$ \\
\hline Siewert $1997[35]$ & CDDP-FU-FA & 41 & $30(73 \%)$ & NS & $56 \%$ \\
\hline Schuhmacher 2001 [36] & EAP & 42 & $31(74 \%)$ & $19 \mathrm{mo}$ & $40 \%$ \\
\hline Ott 2003 [37] & PLF & 42 & $32(76 \%)$ & $25 \mathrm{mo}$ & NS \\
\hline Hartgrink 2004 [38] & FAMTX & 27 & $18(67 \%)$ & $18 \mathrm{mo}$ & $44 \%$ \\
\hline
\end{tabular}

CDDP, cisplatinum; CTx, chemotherapy; EAP, etoposide-adriamycin-cisplatinum; EFP, etoposide-fluorouracil-cisplatin; FAMTX, fluorouracildoxorubicin-methotrexate; FA, folinic acid; FU, 5-fluorouracil; ip, intraperitoneal; iv, intravenous; NS, not specified; PLF = cisplatin-leucovorinfluorouracil

received neoadjuvant chemotherapy [29]. The use of PET in order to assess metabolic response during neoadjuvant chemotherapy for gastric cancer is a very interesting approach but certainly still in a preliminary phase and should be reproduced by larger sample sizes and other investigators [30]. In short, there is as yet no reliable morphological or functional surrogate parameter for response and for the true value of neoadjuvant chemotherapy in gastric cancer. Therefore, the endpoint of neoadjuvant treatment must remain the rate of R0 resections and, more importantly, overall survival.

\section{Results of neoadjuvant chemotherapy}

Phase II studies have demonstrated that neoadjuvant chemotherapy can be given with acceptable toxicity and without excessive postoperative morbidity and mortality (Table 1). However, some adverse effects associated with neoadjuvant treatment, such as chemotherapyinduced anemia necessitating preoperative allogenic red cell transfusion, should be carefully investigated for their prognostic impact [39]. What we know from phase II trials is that patients who respond to neoadjuvant chemotherapy have a markedly improved prognosis compared with nonresponders [36,37,40].

However, until recently, no data from adequately powered randomized studies were available. At the 2003 meeting of the American Society of Clinical Oncology, the first results and preliminary survival analysis from the UK Medical Research Council "MAGIC" trial were presented [2]. In this multi-institutional trial, 503 patients with stage II and III gastric cancer were randomized to receive surgery alone or three cycles of preoperative chemotherapy with epirubicine, cisplatin, and fluorouracil (ECF) followed by surgery followed by three postoperative ECF-cycles. A higher proportion of smaller tumors and of pT1 and pT2 category tumors was found after induction chemotherapy resulting in a higher proportion of potentially curative resections (79\% versus $69 \%, P=0.018, \chi^{2}$ test). Unfortunately, pathologically assessed data on the R classification are unavailable at present. Consequently we do not know, how many resections deemed "curative" by the surgeon later revealed residual microscopic disease (R1). After a median follow-up of 2.0 years, progression-free survival was reported as being significantly prolonged in patients randomized to the perioperative chemotherapy arm $(\log$ rank $P$-value $=0.002$; hazard ratio $=0.7 ; 95 \%$ CI 0.56-0.88). For overall survival, the hazard ratio was 0.8 in favor of chemotherapy, but this difference had not reached significance $(P=0.063)$ at the time of the first evaluation. With a longer follow-up, the survival advantage may become clearer and statistically significant. The 2-year survival rate was $48 \%$ for perioperative chemotherapy versus $40 \%$ for surgery alone. However, one can criticize the inclusion of $11 \%$ patients with cancer of the distal esophagus in this study. This may trouble the interpretation of the results to a certain extent. More importantly, the assessment of the clinical stage lacks routinely performed endoscopic ultrasound and diagnostic laparoscopy. Furthermore, it seems that the surgical quality control was weak. The type of resection was left at the discretion of the participating surgeon, and there was no recommendation given regarding D1 or D2 lymph node dissection.

In summary, the MAGIC trial is the first large randomized trial that strongly supports the efficacy of perioperative chemotherapy in gastric cancer. On the other hand, the trial leaves us with some questions. Therefore, the updated results including the primary endpoint, which is 5-year overall survival, and the results of confirmatory studies should be waited for before one draws definitive conclusions regarding the impact 
of neoadjuvant chemotherapy on the outcome after resection of gastric cancer.

To a certain extent, the results of the MAGIC trial seem to replicate the data obtained in the previously published study of the Medical Research Council Oesophageal Cancer Working Party [41]. Both trials demonstrated an absolute 2-year survival difference in the range of $8 \%$ to $9 \%$. The Medical Research Council (MRC) trial compared surgical resection with or without neoadjuvant chemotherapy in esophageal cancer and revealed a significantly higher number of $\mathrm{R} 0$ resections after preoperative treatment, which translated into a statistically significant difference in the median survival ( 16.8 versus 13.3 months; $P=0.004$ ). It is fair to say that the results from an esophageal cancer trial should not be applied to the problem of gastric cancer without important restrictions. On the other hand, 10\% of the patients included in the MRC trial had a cancer at the cardia and not an esophageal carcinoma. Moreover, the risk reduction by neoadjuvant chemotherapy was particularly high in the group of patients with lowerthird esophageal cancers and with cardia cancers. With regard to survival, only in the subgroup of patients with adenocarcinoma was a statistically significant difference demonstrated. Therefore, although not designed for definitive conclusions concerning adenocarcinomas at the gastroesophageal junction, the results from the MRC trial suggest a clinically meaningful benefit from neoadjuvant chemotherapy for adenocarcinomas at or adjacent to the esophagogastric junction.

\section{Results of adjuvant chemotherapy}

There is wide use of adjuvant chemotherapy after gastric cancer resection in many countries. However, data are not convincing and do not support the general use of this modality in unselected patient populations who underwent curative resection for gastric cancer. One should bear in mind that very few single trials have shown a survival advantage for adjuvant treated patients. In non-Asian countries, only two trials, both limited by the small number of included patients, have shown a significant improvement in survival by adjuvant chemotherapy [42,43]. Meanwhile, several metaanalyses on the effect of adjuvant chemotherapy in gastric cancer have been published [44-47]. As indicated by the odds ratios and the confidence intervals, three of the meta-analyses have demonstrated a very small and statistically borderline significant effect in favor of adjuvant chemotherapy (Table 2). In the most recently published and also largest meta-analysis from the Scandinavian group, this advantage was restricted to the studies carried through in Asia. The cause of this difference is not apparent. Several other restrictions should also be considered. First, many of the trials have a relatively high baseline mortality rate. Therefore, the odds ratio may overestimate the relative risk reduction substantially. Second, the limitations of literature-based meta-analyses in general have to be taken into account. Third, the quality of the literature addressing chemotherapy for gastric cancer is frequently poor with few properly designed randomized trials. In a number of randomized multicenter adjuvant studies the inclusion rates have been remarkably low, which reduces the scientific value of the studies.

The authors of all meta-analyses therefore concluded that there is not sufficient evidence to recommend adjuvant chemotherapy as a routine treatment.

Of note, in one subgroup analysis a trend toward a larger magnitude of the effect of adjuvant chemotherapy was shown when analysis was restricted to trials in which at least two-thirds of patients had nodepositive disease [44]. This finding is interesting, because it is consistent with adjuvant treatments in breast and colon cancer and with effects seen in other studies. For instance, in a randomized Italian multicenter trial a subgroup of patients with a nodal involvement of more than 6 lymph nodes had a clinically meaningful benefit from adjuvant chemotherapy after gastrectomy and D2 lymphadenectomy (5-year survival $42 \%$ versus $22 \%$ ) [48]. Furthermore, both European studies being in favor of adjuvant chemotherapy included node-positive [43] or stage III and therefore mainly node-positive [42] gastric tumors. However, as this finding is based on a subgroup analysis and on two relatively small studies,

Table 2. Meta-analyses on randomized adjuvant chemotherapy studies in gastric cancer

\begin{tabular}{lccc}
\hline $\begin{array}{l}\text { Meta-analysis } \\
\text { (First author; ref. \#) }\end{array}$ & No. studies & No. patients & Odds ratio (CI) \\
\hline Hermans 1993 [46] & 11 & 2096 & $0.88(0.78-1.08)$ \\
Earle 1999 [45] & 13 & 1990 & $0.80(0.66-0.97)$ \\
Mari 2000 [47] & 21 & 3658 & $0.82(0.75-0.89)$ \\
Janunger 2002 [44] & 21 & 3962 & $0.84(0.74-0.96)$ \\
\hline
\end{tabular}

CI, confidence interval 
respectively, it can only serve to generate hypotheses and should be studied in a large prospective randomized trial. Nevertheless, it suggests that patients at higher risk for recurrence may be the ones who benefit most from adjuvant chemotherapy. Future trials might increase their efficiency by focussing on this group of patients. The right selection could be based on modern prognostic nomograms like the New York Memorial Sloan Kettering Cancer Center Score [49] or on the ratio of the number of invaded in relation to the number of removed lymph nodes, which proved to be of prognostic significance in a multicenter trial carried out in Germany [7].

\section{Results of adjuvant chemoradiation}

After the publication of the SWOG9008/INT0116 study, adjuvant chemoradiation after curative gastric resection became a standard of care in some countries. Adjuvant therapy consisted of five cycles of 5fluorouracil given intravenously as a bolus plus leucovorin plus $45 \mathrm{~Gy}$ of external beam radiation at $1.8 \mathrm{~Gy}$ per day, given 5 days per week together with the dosereduced second and third cycles of chemotherapy [3]. At first glance, the statistically significant survival advantage in the adjuvant treatment arm would justify the general use of this adjuvant treatment (Table 3). However, one has to keep in mind that the results were obtained from surgically undertreated patients. Although the study protocol recommended D2 lymphadenectomy based on the resection of the second-tier lymph nodes according to the AJCC TNM classification, which at that time was very similar to the Japanese stage classification, and included appropriate instructions, $54 \%$ of the study patients underwent a "less than D1" lymphadenectomy. As reported in a second publication by the same group of authors, surgical undertreatment clearly undermined survival [50]. Therefore, one might presume that adjuvant chemoradiation did no more than compensate to a certain extent for the sequels of inadequate surgery. While on the one side the absolute number of local relapses (51 patients after surgery alone versus 23 patients after surgery plus chemoradiation) and regional relapses (127 versus 78 patients) was higher in the surgery alone group, on the other side no difference was observed in the number of distant relapses (32 versus 40 patients). The improvement in survival after chemoradiation observed in this study therefore arises solely from a better locoregional tumor control. Compared to other series, the magnitude of locoregional relapses seems to be particularly high in this trial. As it has become clear now that extended lymphadenectomy leads to better tumor control and survival [51], one might assume that after D2 lymphadenectomy, adjuvant chemoradiation would add little or nothing to the patients' prognosis. Therefore, the efficacy of adjuvant chemoradiation should absolutely be reproduced in a quality-controlled multicenter study which includes patients after D2 lymphadenectomy and which has a control arm without postoperative adjuvant treatment.

Another matter of concern is the limited tolerability of adjuvant chemoradiation. The SWOG9008/INT0116 study included only patients deemed fit for adjuvant therapy. There is no information at this point on how many patients could not be enrolled due to prolonged postoperative recovery and due to typical postsurgical complications. However, of the 281 patients assigned to the chemoradiotherapy group, only 181 (64\%) completed treatment as planned. This drop-out rate exceeds by far the numbers usually seen during adjuvant chemotherapy protocols in many malignancies. The most commonly severe toxicity was reported to be hematologic (54\% of patients) and gastrointestinal (33\%) side effects. It was frequently brought up that the relatively large radiation fields being applied in the SWOG9008/ INT0116 study may account for the mediocre tolerance of the treatment. However, even with modern strategies of adjuvant chemoradiation, toxicity remains considerable. Weight loss is a considerable concern in this group of patients [52]. Recently presented data suggest that late

Table 3. Results of the randomized phase III "SWOG 9008/INT 0116" trial assessing the role of adjuvant chemoradiation after curative gastric cancer resection compared to surgery alone [3]

\begin{tabular}{|c|c|c|c|}
\hline & $\begin{array}{l}\text { Adjuvant } \\
(n=281)\end{array}$ & $\begin{array}{l}\text { Surgery alone } \\
\quad(n=275)\end{array}$ & $P$ value \\
\hline Median duration of survival & 36 months & 27 months & 0.005 \\
\hline Three-year survival rate & $50 \%$ & $41 \%$ & \\
\hline Hazard ratio for death & 1.0 & 1.35 (95\% CI 1.09-1.66) & 0.005 \\
\hline Median relapse-free survival & 30 months & 19 months & 0.001 \\
\hline Three-year relapse-free survival & $48 \%$ & $31 \%$ & \\
\hline Hazard ratio for relapse & 1.0 & $1.52(95 \%$ CI $1.23-1.86)$ & 0.001 \\
\hline
\end{tabular}

$\overline{\text { SWOG9008/INT0116, Southwest Oncology Group 9008/Intergroup 0116; CI, confidence interval }}$ 
renal toxicity after postoperative chemoradiotherapy in gastric cancer also merits thorough attention [53].

In summary, the controversial role of adjuvant chemoradiation in gastric cancer is still being discussed. Based on the findings of the SWOG9008/Intergroup0116 trial, postoperative chemoradiation should only be recommended when the surgical objective of an extended (D2) lymphadenectomy is missed. The situation of microscopically incomplete resection (R1) has not been sufficiently studied to draw definitive conclusions.

\section{Nonmetastatic gastric cancer: suggestion for a multimodal treatment approach}

As data from the literature supporting multimodal strategies are not persuasive at this stage, clinically staged standard risk gastric cancer should be resected (D2 lymphadenectomy) and, in the absence of risk factors apparent from the pathological report, patients should not routinely undergo adjuvant treatment. This procedure should also be the control arm of new clinical trials evaluating the role of specific pre- or postoperative interventions. But in the case of a positive survival endpoint reported by the "MAGIC" investigators, neoadjuvant chemotherapy should be considered in all stage II and stage III tumors.
In cancers with a limited probability of a complete (R0) resection, i.e., endoscopically staged uT3 and uT4 tumors, it can already be recommended at present that neoadjuvant chemotherapy be administered. The sensitivity to chemotherapy can best be judged from the histomorphological grading of regression obtained after preoperative treatment, when the patient underwent resection [54].

At our clinic, the recommendation for or against postoperative, adjuvant treatment is based on the response to neoadjuvant treatment, on the presence of pathological risk factors, i.e., the extent of lymph node involvement, and on the completeness of the surgical resection including a D2 lymphadenectomy, being judged appropriate when at least 25 lymph nodes are removed (Fig. 1).

\section{Perspectives}

One major goal of future clinical research will be to get a clearer definition of prognostic and predictive markers. This should help to customize adjuvant and neoadjuvant treatment strategies for the individual patient.

One approach is based on biochemical technologies. Detection of tumor targets or molecules associated with the mechanisms of action of anticancer drugs may pre-

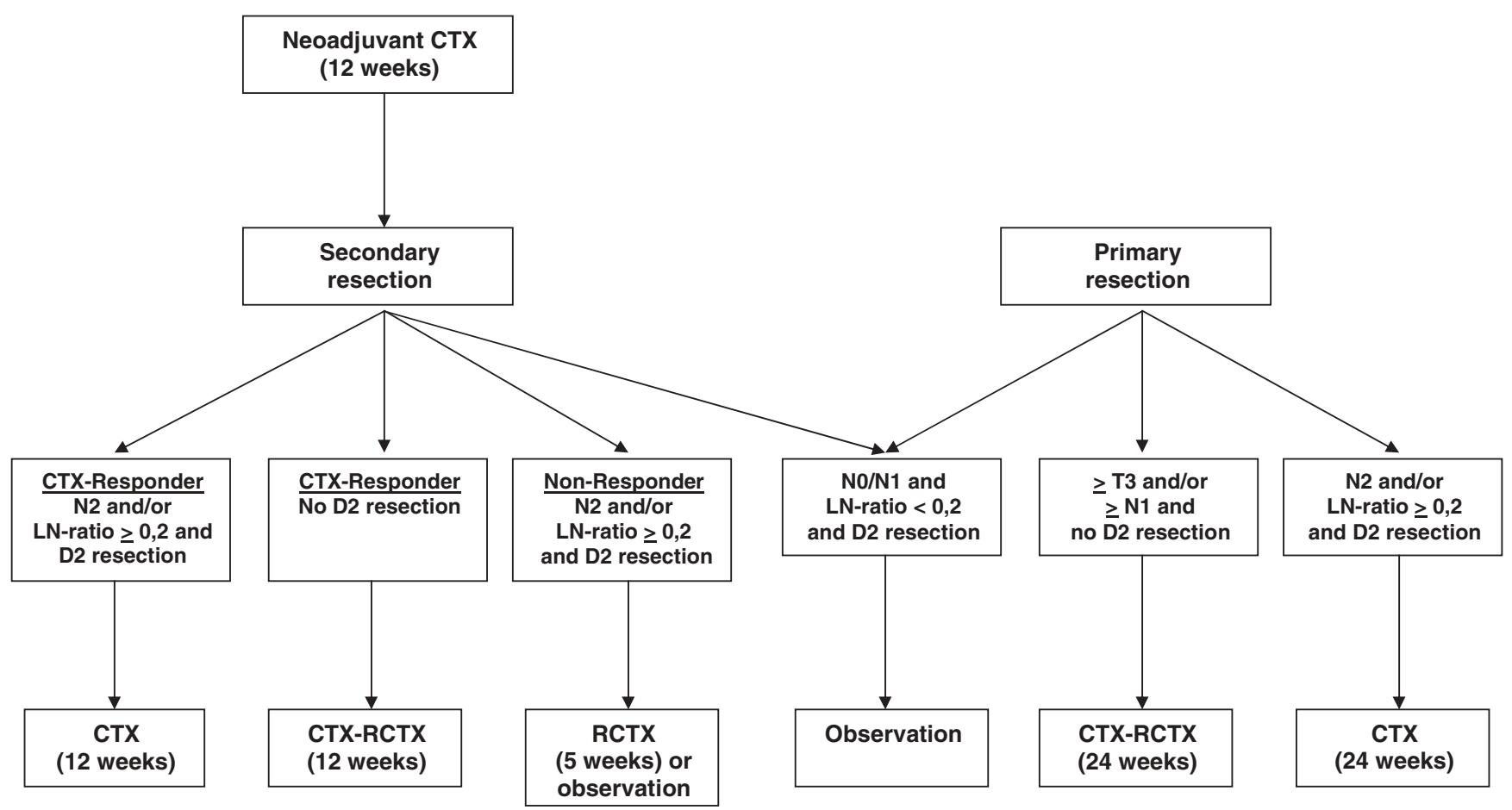

Fig. 1. Individualized recommendation for adjuvant treatment strategies in gastric cancer patients who underwent neoadjuvant chemotherapy or those who underwent primary resection. CTX, Chemotherapy; LN-ratio, ratio between invaded and removed lymph nodes; RCTX, radiochemotherapy 
dict the likelihood of treatment response. Expression of 5-fluorouracil-related genes or enzymes such as the tumor thymidilate synthase, thymidine phosphorylase, and dipyrimidine dehydrogenase, and of cisplatinrelated genes such as ERCC1 or GADD45 $\alpha$ may help to predict response to chemotherapy containing 5fluorouracil and cisplatin [55-58]. However, it must be emphasized that these findings have not yet been validated in large-scale studies and therefore cannot serve as a basis for clinical decision making at this stage.

An alternative approach is the early assessment of metabolic changes in the tumor by means of molecular imaging techniques such as PET. One such model is the quantification of changes in the 5-FDG uptake into tumor tissue. Two studies have demonstrated that PET imaging may differentiate responding and nonresponding tumors early in the course of neoadjuvant systemic chemotherapy [30,59].

With the expansion of our chemotherapeutic armamentarium by compounds such as oxaliplatin, irinotecan, and docetaxel, all of which have shown promising activity in the treatment of gastric cancer, there is certainly a need for new, more active, or better tolerated induction chemotherapy protocols to be tested in phase II and phase III studies. Probably, the introduction of new biologically targeted compounds will further improve the efficacy of adjuvant and neoadjuvant treatment.

Presumably, the future will bring tailored treatment strategies based on the reliable specification of constitutional and tumor-related characteristics. As indicated by preliminary data [60], genomic profiling may be a key technology in this context. It is hoped that a better understanding of the individual tumor biology will make treatments more effective and will reduce overand undertreatment of patients with gastric cancer.

\section{References}

1. Hundahl SA, Menck HR, Mansour EG, Winchester DP. The National Cancer Data Base report on gastric carcinoma. Cancer 1997;80:2333-41.

2. Allum W, Cunningham D, Weeden $S$ on behalf of the NCRI Upper GI Clinical Study Group. Perioperative chemotherapy in operable gastric and lower oesophageal cancer. A randomised controlled trial (the MAGIC trial ISRCTN 93793971); Proc Am Soc Clin Oncol 2003;998a.

3. Macdonald JS, Smalley SR, Benedetti J, Hundahl S, Estes NC, Stemmermann GN, et al. Chemoradiotherapy after surgery compared with surgery alone for adenocarcinoma of the stomach or gastroesophageal junction. N Engl J Med 2001;345:725-30.

4. Bonenkamp JJ, Hermans J, Sasako M, Van de Velde CJH for the Dutch Gastric Cancer Group. Extended lymph-node dissection for gastric cancer. N Engl J Med 1999;340:908-14.

5. Hartgrink HH, van de Velde CJH, Putter H, Bonenkamp JJ, Klein Kranenbarg E, Songun I, et al. Extended lymph node dissection for gastric cancer: who may benefit? Final results of the Randomized Dutch Gastric Cancer Group Trial. J Clin Oncol 2004;22:2069-77.
6. Mcculloch P, Eidi Nita M, Kazi H, Tsuno H, Nagawa H, GamaRodrigues JJ. Extended versus limited lymph nodes dissection technique for adenocarcinoma of the stomach. Cochrane Database Syst Rev 2003;4:CD001964.

7. Siewert JR, Böttcher K, Stein HJ, Roder JR and the German Gastric Carcinoma Study Group. Relevant prognostic factors in gastric cancer. Ann Surg 1998;228:449-61.

8. Maruyama K, Sasako M, Kinoshita T, Sano T, Katai H, Hada M, et al. Current controversies in cancer: should systematic lymph node dissection be recommended for gastric cancer? Eur J Cancer 1998;34:1480-9.

9. Van de Velde CJH, Peeters KCMJ. The gastric cancer controversy. J Clin Oncol 2003;21:2234-6.

10. Siewert JR, Feith M, Werner M, Stein HJ. Adenocarcinoma of the esophagogastric junction: results of surgical therapy based on anatomical/topographic classification in 1002 consecutive patients. Ann Surg 2000;232:353-61.

11. Mariette C, Castel B, Toursel H, Fabre S, Balon JM, Triboulet JP. Surgical management of and long-term survival after adenocarcinoma of the cardia. Br J Surg 2002;89:1156-63.

12. Stein HJ, Feith M, Siewert JR. Cancer of the esophago-gastric junction. Surg Oncol 2000;9:35-41.

13. Sasako M, Sano T, Sairenji M, Arai T, Kinoshita T, Yamamoto S, et al. Left thoraco-abdominal approach (LT) compared with abdominal and transhiatal approach (AT) for cardia or subcardia cancer. Results of a surgical randomized controlled trial (JCOG9502). J Clin Oncol 2004 ASCO Annual Meeting Proceedings;22(Suppl 14S):4000(abstract).

14. Rösch T. Endosonographic staging of gastric cancer: a review of literature results. Gastrointest Endosc Clin N Am 1995;5:549-57.

15. Dittler HJ. Assessment of resectability of gastrointestinal cancers by endoscopic ultrasonography. Gastrointest Endosc Clin N Am 1995;3:569-75.

16. Willis S, Truong S, Gribnitz S, Fass J, Schumpelick V. Endoscopic ultrasonography on the preoperative staging of gastric cancer. Accuracy and impact on surgical therapy. Surg Endosc 2000; 14:951-4.

17. Lehnert T, Rudek B, Kienle P, Buhl K, Herfarth C. Impact of diagnostic laparoscopy on the management of gastric cancer: prospective study of 120 consecutive patients with primary gastric adenocarcinoma. Br J Surg 2002;89:471-5.

18. Feussner H, Omote K, Fink U, Walker SJ, Siewert JR. Pretherapeutic laparoscopic staging in advanced gastric carcinoma. Endoscopy 1999;31:342-7.

19. Hayes N, Wayman J, Wadehra V, Scott DJ, Raimes SA, Griffin SM. Peritoneal cytology in the surgical evaluation of gastric carcinoma. Br J Canc 1999;79:520-4.

20. Nakajima T, Harashima S, Hirata M, Kajitani T. Prognostic and therapeutic values of peritoneal cytology in gastric cancer. Acta Cytol 1978;22:225-9.

21. Nekarda H, Geß C, Stark M, Mueller JD, Fink U, Schenck U, et al. Immunocytochemically detected free peritoneal tumour cells (FPTC) are a strong prognostic factor in gastric carcinoma. Br J Canc 1999;79:611-9.

22. De Potter T, Flamen P, van Cutsem E, Penninckx F, Filez L, Bormans A, et al. Whole-body PET with FDG for the diagnosis of recurrent gastric cancer. Eur J Nucl Med 2002;29:525-9.

23. Stahl A, Ott K, Weber WA, Becker K, Link T, Siewert, et al. PET imaging of locally advanced gastric carcinomas: correlation with endoscopic and histopathological findings. Eur J Nucl Med Mol Imaging 2003;30:288-95.

24. Yu W, Seo BY, Chung HY. Postoperative body-weight loss and survival after curative resection for gastric cancer. Br J Surg 2002; 89:467-70.

25. Macadam R, Sarela A, Wilson J, MacLennan K, Guilou P. Bone marrow micrometastases predict early post-operative recurrence following surgical resection of oesophageal and gastric carcinoma. Eur J Surg Oncol 2003;29:450-4.

26. Landry J, Tepper JE, Wood WC, Moulton EO, Koerner F, Sullinger J. Patterns of failure following curative resection of 
gastric carcinoma. Int J Radiat Oncol Biol Phys 1990;19:135762 .

27. Tsuburaya A, Sasako M, Furukawa A, Fukushima M, Fujitani T, Tanemura $\mathrm{H}$, et al. Preoperative adjuvant chemotherapy with irinotecan and cisplatin for gastric cancer with extensive lymph node metastasis: a multicenter phase II study by Japan Clinical Oncology Group (JCOG0001). Proc ASCO Gastrointestinal Cancer Symposium 2005:3(abstr).

28. Therasse P, Arbuck SG, Eisenhauer EA, Wanders J, Kaplan RS, Rubinstein L, et al. New guidelines to evaluate the response to treatment in solid tumors. J Nat Canc Inst 2000;92:205-16.

29. Kelsen D, Karpeh M, Schwartz G, Gerdes H, Lightdale C, Botet $\mathrm{J}$, et al. Neoadjuvant therapy of high-risk gastric cancer: a phase II trial of preoperative FAMTX and postoperative intraperitoneal fluorouracil-cisplatin plus intravenous fluorouracil. J Clin Oncol 1996;14:1818-28.

30. Ott K, Fink U, Becker K, Stahl A, Dittler HJ, Busch R, et al. Prediction of response to preoperative chemotherapy in gastric carcinoma by metabolic imaging: results of a prospective trial. J Clin Oncol 2003;21:4604-10.

31. Wilke H, Preusser P, Fink U, Gunzer U, Meyer HJ, Meyer J, et al. Preoperative chemotherapy in locally advanced and nonresectable gastric cancer: a phase II study with etoposide, doxorubicin and cisplatin. J Clin Oncol 1989;7:1318-26.

32. Ajani JA, Ota DM, Jessup JM, Ames FC, McBride C, Boddie A, et al. Resectable gastric carcinoma. An evaluation of preoperative and postoperative chemotherapy. Cancer 1991;68:1501-6.

33. Alexander HR, Grem JL, Pass HI, Hamilton M, McAtee N, Fraker DL, et al. Neoadjuvant chemotherapy for locally advanced gastric adenocarcinoma. Oncology (Huntington) 1993;7:3742.

34. Crookes P, Leichman CG, Leichman L, Tan M, Laine L, Stain S, et al. Sytemic chemotherapy for gastric carcinoma followed by postoperative intraperitoneal therapy. Cancer 1997;79:176775 .

35. Siewert JR, Fink U, Sendler A, Becker K, Bottcher K, Feldmann H, et al. Gastric cancer. Curr Probl Surg 1997;34:835-9.

36. Schuhmacher CP, Fink U, Becker K, Busch R, Dittler HJ, Mueller J, et al. Neoadjuvant therapy for patients with locally advanced gastric carcinoma with etoposide, doxorubicin, and cisplatinum. Cancer 2001;91:918-27.

37. Ott K, Sendler A, Becker K, Dittler HJ, Helmberger H, Busch R, et al. Neoadjuvant chemotherapy with cisplatin, 5-FU, and leucovorin (PLF) in locally advanced gastric cancer: a prospective phase II study. Gastric Cancer 2003;6:159-67.

38. Hartgrink HH, van de Velde CJH, Putter H, Songun I, Tesselaar MET, Klein Kranenbarg E, et al. Neo-adjuvant chemotherapy for operable gastric cancer: long term results of the Dutch randomised FAMTX trial. Eur J Surg Oncol 2004;30:643-9.

39. Voelter V, Schuhmacher C, Busch R, Peschel C, Siewert JR, Lordick F. Incidence of anemia in patients receiving neoadjuvant chemotherapy for locally advanced esophagogastric patients. Ann Thorac Surg 2004;78:1037-41.

40. Lowy AM, Mansfield PF, Leach SD, Pazdur R, Dumas P, Ajani JA. Response to neoadjuvant chemotherapy best predicts survival after curative resection for gastric cancer. Ann Surg 1999; 229:303-8.

41. Medical Research Council Oesophageal Cancer Working Party. Surgical resection with or without preoperative chemotherapy in oesophageal cancer: a randomised controlled trial. Lancet 2002;359:1727-33.

42. Cirera L, Balil A, Batiste-Alentorn E, Tusquets I, Cardona T, Arcusa A, et al. Randomized clinical trial of adjuvant mitomycib plus tegafur in patients with resected stage III gastric cancer. J Clin Oncol 1999;17:3810-5.

43. Neri B, Cini G, Andreoli F, Boffi B, Francesconi D, Mazzantini R, et al. Randomized trial of adjuvant chemotherapy versus control after curative resection for gastric cancer: 5-year follow-up. Br J Cancer 2001;84:878-80.
44. Janunger KG, Hafstrom L, Glimelius B. Chemotherapy in gastric cancer: a review and updated meta-analysis. Eur J Surg 2002; 168:597-608.

45. Earle CC, Maroun JA. Adjuvant chemotherapy after curative resection for gastric cancer in non-Asian patients: revisiting a meta-analysis of randomised trials. Eur J Cancer 1999;35:1059-64.

46. Hermans J, Bonenkamp JJ, Boon MC, Bunt AM, Ohyama S, Sasako M, et al. Adjuvant therapy after curative resection for gastric cancer: meta-analysis of randomized trials. J Clin Oncol 1993;11:1441-7.

47. Mari E, Floriani I, Tinazzi A, Buda A, Belfiglio M, Valentini M, et al. Efficacy of adjuvant chemotherapy after curative resection for gastric cancer: A meta-analysis of published randomised trials. Ann Oncol 2000;11:837-43.

48. Bajetta E, Buzzoni R, Mariani L, Beretta E, Bozzetti F, Bordogna $\mathrm{G}$, et al. Adjuvant chemotherapy in gastric cancer: 5-year results of a randomised study by the Italian Trials in Medical Oncology (ITMO) Group. Ann Oncol 2002;13:299-307.

49. Kattan MW, Karpeh MS, Mazumdar M, Brennan MF. Postoperative nomogram for disease-specific survival after R0 resection for gastric carcinoma. J Clin Oncol 2003;21:3647-50.

50. Hundahl SA, Macdonald JS, Benedetti J, Fitzsimmons T for the Southwest Oncology Group and the Gastric Intergroup. Surgical treatment variation in a prospective, randomized trial of chemoradiotherapy in gastric cancer: the effect of undertreatment. Ann Surg Oncol 2002;9:278-86.

51. Schwarz RE, Smith DD. Clinical impact of lymphadenectomy extent in resectable, advanced-stage gastric cancer. Proc ASCO Gastrointestinal Cancers Symposium 2005:85 (abstr 6).

52. Bokemeyer C, Budach W, Stahl M, Vanhoefer U, Hehr T, Wilke J, et al. Adjuvant radiochemotherapy (RTx/CTx) using 5-FU/ Folinic acid (FA)/cisplatin (DDP) $+/-$ Paclitaxel (P) and radiation in patients (pts) with completely (R0) resected high-risk gastric cancer (UICC stages II-IV(M0): An extended phase II study of the AIO/ARO/ACO. Ann Oncol 2004;15 (Suppl.3):iii231 (abstr 878PD)

53. Verheij M, Oppedijk V, Boot H, Cats A, Saunders M, Khoo V, et al. Late renal toxicity following post-operative chemoradiotherapy in gastric cancer. Proc ASCO Gastrointestinal Cancers Symposium 2005:83 (abstr 2).

54. Becker K, Mueller JD, Schulmacher C, Ott K, Fink U, Busch R, et al. Histomorphology and grading of regression in gastric carcinoma treated with neoadjuvant chemotherapy. Cancer 2003;98: 1521-30.

55. Boku N, Chin K, Hosokawa K, Ohtsu A, Tajiri H, Yoshida S, et al. Biological markers as a predictor for response and prognosis of unresectable gastric cancer patients treated with 5-fluorouracil and cis-platinum. Clin Cancer Res 1998;4:1469-74.

56. Lenz HJ, Leichman CG, Danenberg K, Danenberg PV, Groshen $\mathrm{S}$, Cohen $\mathrm{H}$. Thymidylate synthase mRNA level in adenocarcinoma of the stomach. A predictor for primary tumor response and overall survival. J Clin Oncol 1996;14:176-82.

57. Metzger R, Leichman CG, Danenberg KD, Danenberg PV, Lenz HJ, Hayashi K et al. ERCC1 mRNA levels complement thymidylate synthase mRNA levels in predicting response and survival for gastric cancer patients receiving combination cisplatin and fluorouracil chemotherapy. J Clin Oncol 1998;16:309-16.

58. Napieralski R, Ott K, Kremer M, Specht K, Vogelsang H, Becker $\mathrm{K}$, et al. Combined GADD45 $\alpha$ and TP expression levels are associated with response and survival of advanced gastric cancer patients after a cisplatin and 5FU based preoperative chemotherapy. Clin Canc Res 2005; in press.

59. Weber WA, Ott K, Becker K, Dittler HJ, Helmberger H, Avril $\mathrm{NE}$, et al. Prediction of response to preoperative chemotherapy in adenocarcinomas of the esophagogastric junction by metabolic imaging. J Clin Oncol 2001;19:3058-65.

60. Weiss MM, Kuipers EJ, Postma C, Snijders AM, Siccama I, Pinkel $\mathrm{D}$, et al. Genomic profiling of gastric cancer predicts lymph node status and survival. Oncogene 2003;22:1872-9. 